\title{
Artikel
}

Cyrill P. Rigamonti*

\section{Walter Benjamin und das Urheberrecht. Zur Unterscheidung zwischen Original und Kopie}

Dieser Beitrag basiert auf zwei Vorträgen zum gleichen Thema, die der Autor anlässlich eines Workshops des Walter Benjamin Kollegs der Universität Bern sowie im Rahmen einer Sommerakademie der Schweizerischen Studienstiftung hielt. Dank gebührt Rechtsanwältin Marie Baumann, LL.M., für ihre Unterstützung bei der Fertigstellung dieses Beitrags. 
Abstract: Dieser Beitrag befasst sich mit der Bedeutung der Unterscheidung zwischen Original und Kopie im modernen Urheberrecht und untersucht ausgewählte gesetzliche Regeln, die mit dieser Unterscheidung operieren. In diesem Zusammenhang wird auch der Frage nachgegangen, ob und inwiefern sich das geltende Recht heute noch an dem von Walter Benjamin geprägten Verständnis des Kunstwerks im Zeitalter seiner technischen Reproduzierbarkeit orientiert.

Keywords: Original, Kopie, Urheberrecht, Copyright, Walter Benjamin, Aura, Originalwerkexemplar, Zerstörungsschutz, Folgerecht, Kunstwerk, Reproduktion

* Prof. Dr. iur. Cyrill P. Rigamonti, Rechtsanwalt, LL.M., S.J.D., Universität Bern, Institut für Wirtschaftsrecht, Schanzeneckstrasse 1, CH-3001 Bern, email: cyrill.rigamonti@iwr.unibe.ch

\section{Einleitung}

Walter Benjamin ist einer der wenigen nichtjuristischen Autoren, die in der juristischen Literatur immer wieder zitiert werden. ${ }^{1}$ Wenig überraschend steht dabei sein berühmtes Essay zum "Kunstwerk im Zeitalter seiner technischen Reproduzierbarkeit" im Vordergrund. ${ }^{2}$ Innerhalb der Rechtswissenschaften sind die Ausführungen Benjamins zur Reproduktion von Kunstwerken und zur ,Aura' des Originals primär für das Urheberrecht von Interesse, ${ }^{3}$ denn es ist gerade das Urheberrecht (,copyright law'), das - unter gewissen Voraussetzungen - Kunstwerke gegen Kopien Dritter schützt. ${ }^{4}$ Entsprechend reizvoll ist es, die

1 Siehe z.B. Mosimann / Müller-Chen 2011, S. 1303; Jayme 2016, S. 21; Dreier 2017, S. 135.

2 In diesem Beitrag wird die letzte von Benjamin autorisierte Fassung verwendet, die bisweilen auch als, dritte Fassung' bezeichnet wird (Benjamin 1991, S. 471-508). Zur komplexen Redaktionsgeschichte, siehe Schöttker 2015, S. 118-133.

3 Vgl. auch Jayme 2016, S. 21, der in Benjamin eine der "geistigen Wurzeln" für die Übernahme des Originalbegriffs im Urheberrecht sieht.

4 Damit sei freilich nicht gesagt, dass die Unterscheidung zwischen Original und Kopie nicht auch in anderen Rechtsgebieten relevant sein kann, aber die spezifische Verknüpfung dieser Unterscheidung mit den Konzepten Walter Benjamins findet sich praktisch ausschließlich in urheberrechtlichem Kontext.
Unterscheidung zwischen Original und Kopie, wie sie in Benjamins Gegenüberstellung von Kunstwerk und Reproduktion zum Ausdruck kommt, ${ }^{5}$ einer näheren urheberrechtlichen Analyse zu unterziehen. Zugleich soll in diesem Beitrag der Frage nachgegangen werden, ob und inwiefern sich die Konzepte Walter Benjamins im modernen Urheberrecht niedergeschlagen haben. ${ }^{6} \mathrm{Um}$

5 Benjamin verwendet die Begriffe ,Kunstwerk' und ,Original' synonym (siehe z.B. Benjamin 1991, S. 475-476: "Hier und Jetzt des Kunstwerks" bzw. "Hier und Jetzt des Originals"). Demgegenüber scheint er über weite Strecken die ,Reproduktion' grundsätzlich als Unterbegriff der ,Kopie' zu verstehen, und zwar in dem Sinne, dass mit Reproduktionen nur, aber immerhin, ,technisch' hergestellte Kopien gemeint sind. Diesen Unterschied hebt Benjamin allerdings in zumindest einer Passage gleich selbst wieder auf, wenn er sowohl von ,technischen' als auch von ,manuellen' Reproduktionen spricht (Benjamin 1991, S. 475-476 und Fn. 2); zur kunsthistorisch diffizilen Unterscheidung zwischen Reproduktion und Kopie, siehe auch Hamann 1949, S. 135-139. Diese partielle terminologische Ambivalenz kann vorliegend indes vernachlässigt werden, weil es urheberrechtlich ohnehin keine Rolle spielt, wie eine ,Kopie' oder eine ,Reproduktion' hergestellt wird. Es wird hier demnach der Einfachheit halber davon ausgegangen, dass die Unterscheidung zwischen Kunstwerk und Reproduktion bei Benjamin der Unterscheidung zwischen Original und Kopie entspricht.

6 Weil es, das' Urheberrecht nicht gibt, sondern primär eine Vielzahl nationaler Urheberrechte, sei an dieser Stelle klargestellt, dass sich die nachstehenden Ausführungen primär auf das schweizerische und deutsche Urheberrecht unter 
meine Ausführungen interdisziplinär anschlussfähig zu halten, habe ich mich bemüht, möglichst wenige juristische Kenntnisse vorauszusetzen die Fachwelt möge es mir verzeihen.

\section{Urheberrechtliche Leitdifferenz: Werk und Werkexemplar}

Bevor auf die urheberrechtliche Bedeutung der Unterscheidung zwischen Original und Kopie eingegangen werden kann, ist als Ausgangspunkt vorab klarzustellen, dass die Leitdifferenz im Urheberrecht - um einen Begriff aus der Systemtheorie ${ }^{7}$ fachfremd zu verwenden - nicht die Unterscheidung zwischen Original und Kopie ist, sondern die Unterscheidung zwischen Werk und Werkexemplar. ${ }^{8}$

Dies liegt daran, dass sich das Urheberrecht mit Werken der Literatur und Kunst befasst, also mit geistigen Schöpfungen (,works of authorship'). ${ }^{9}$ Geistige Schöpfungen sind immaterielle Güter, und Urheberrechte daran sind daher stets Rechte an unkörperlichen Gegenständen. ${ }^{10}$ Werke als Gegenstand des Urheberrechts sind strikte zu unterscheiden von Werkexemplaren, also denjenigen physischen Objekten, die das geistige Werk verkörpern. Es handelt sich dabei rechtlich nicht um Werke, sondern um Sachen, an denen grundsätzlich keine Urheberrechte bestehen, sondern Eigentum. Der Eigentumsbegriff ist heute in den

Einschluss des Rechts der Europäischen Union und unter Berücksichtigung des amerikanischen Rechts beziehen.

7 Luhmann 1987, S. 19, 57, 105.

8 Anstelle von, Werkexemplar' wird bisweilen auch der Begriff ,Werkstück' verwendet; siehe z.B. Rehbinder 2000, Rz. 39-40, S. 57-58. Urheberrechtlich handelt es sich dabei um Synonyme.

9 Siehe z.B. Art. 1 Abs. 1 lit. a i.V.m. Art. 2 Abs. 1 des schweizerischen Urheberrechtsgesetzes (hiernach "URG") oder $\S \S 1$ und 2 Abs. 2 des deutschen Urheberrechtsgesetzes (hiernach „UrhG").

10 Urheberrechtsgesetze können den Rechtsinhabern freilich auch Rechte zugestehen, die keine Rechte an immateriellen Gütern sind, z.B. Zutrittsrechte zu bestimmten physischen Objekten, die urheberrechtliche Werke verkörpern; siehe Art. 14 URG oder § 25 UrhG. Dabei handelt es sich juristisch indes nicht um Urheberrechte im materiellen Sinn, eben weil es nicht um Rechte an Werken geht, sondern um gesetzliche Realobligationen, also um relative Rechte an Sachen; siehe dazu Rigamonti 2013, S. 324. meisten Rechtsordnungen auf absolute Rechte an körperlichen Gegenständen beschränkt.

Die Unterscheidung zwischen Werk und Werkexemplar ist grundlegend und einfach zu verstehen. Das literarische Werk wird im Buch verkörpert. Am literarischen Werk als geistige Schöpfung können Urheberrechte bestehen, und am Buch als körperlicher Gegenstand besteht Eigentum. Wer ein Buch kauft, erwirbt Eigentum an diesem bestimmten physischen Werkexemplar, erwirbt dadurch aber keine Urheberrechte am potentiell darin verkörperten literarischen Werk.

Im vorliegenden Zusammenhang ist festzuhalten, dass sich die urheberrechtliche Leitdifferenz zwischen Werk und Werkexemplar nicht mit der Unterscheidung zwischen Original und Kopie deckt. Es ist gerade nicht so, dass das Werk als geistige Schöpfung das ,Original' und das Werkexemplar als Verkörperung dieses Werks die ,Kopie' darstellt. Wenn eine Künstlerin eine unikale Skulptur schafft, dann wird kaum jemand dieses Unikat als Kopie bezeichnen, sondern eben als Original, obwohl urheberrechtlich immer noch zwischen dem Werk als geistiger Schöpfung und dem Werkexemplar als Verkörperung dieser Schöpfung unterschieden wird.

Auf den ersten Blick scheint dies in Rechtsordnungen wie der amerikanischen grundsätzlich anders zu sein, weil dort der Begriff der Kopie (,copy') inhaltlich gerade als Werkexemplar definiert wird (",copies are material objects [...] in which a work is fixed") ${ }^{11}$ und Werke überdies als "original works of authorship" verstanden werden. ${ }^{12}$ Bei näherer Betrachtung zeigt sich jedoch, dass der hier als Adjektiv verwendete Ausdruck, original' gerade nicht das Werk als solches bezeichnet, sondern die Voraussetzung nennt, die gegeben sein muss, damit ein Werk urheberrechtlich geschützt ist. International ist in diesem Zusammenhang abgesehen von Origi-

1117 U.S.C. § 101. Mit Blick auf diese gesetzlichen Begriffsvorgaben nicht vollends zu überzeugen vermag die von Dreier gerade im Kontext des amerikanischen Rechts verwendete Terminologie, die - anders als Benjamin (siehe dazu oben Fn. 5) - den Begriff der Reproduktion dem Begriff des Werkexemplars gleichstellt und dann innerhalb der Reproduktionen zwischen Originalen und Kopien unterscheidet; siehe Dreier 2016, S. 254-255. Für den deutschsprachigen Raum trifft Dreiers Definition von Original und Kopie hingegen zu.

1217 U.S.C. § 102(a). 
nalität auch von Individualität oder Einmaligkeit die Rede. Um die Gegenüberstellung von Original und Kopie, wie Walter Benjamin die Unterscheidung zwischen Kunstwerk und Reproduktion versteht, geht es aber auch hier nicht.

Wenn die Unterscheidung von Original und Kopie nach dem Gesagten nicht einfach die urheberrechtliche Leitdifferenz zwischen Werk und Werkexemplar abbildet, dann bleibt immer noch die Möglichkeit, die Unterscheidung auf der einen oder der anderen Seite der Leitdifferenz anzuwenden, indem der Begriff des Originals in der technischen Sprache des Urheberrechts entweder als ,Originalwerk' oder als ,Originalwerkexemplar' verstanden wird. Wie sogleich zu zeigen sein wird, findet man bei Walter Benjamin beide Varianten, auch wenn er selbst wohl die zweite Variante bevorzugt haben dürfte.

\section{Original als Originalwerk?}

Verortet man die Unterscheidung von Original und Kopie auf der Werkseite der urheberrechtlichen Leitdifferenz, dann bezeichnen zwangsläufig beide Begriffe, Werke' im Sinne geistiger Schöpfungen. Die Differenz von Original und Kopie dient in diesem Fall dazu, zwischen verschiedenen Werken zu unterscheiden. Mit dem Begriff ,Original' wäre dann ein bestimmtes, urheberrechtlich geschütztes Ausgangswerk gemeint, während der Begriff der ,Kopie' eine andere geistige Schöpfung bezeichnen würde, die dem ,Original' so ähnlich ist, dass sie in dessen Schutzbereich fällt und entsprechend vom Urheberrecht am Original erfasst würde. Dabei spielt es auf der Werkseite der Leitdifferenz selbstredend keine Rolle, ob die so verstandene Kopie in einem Werkexemplar (z.B. einem Buch) verkörpert ist oder ob sie lediglich ephemer ist (z.B. bei einem Vortrag), ${ }^{13}$ denn es geht ja um den Vergleich zweier geistiger Schöpfungen.

Diese Art der Verwendung des Begriffspaars klingt auch bei Walter Benjamin an, denn bekanntlich beginnt er sein Essay gerade mit der Feststellung, dass das Kunstwerk grundsätzlich

13 Nach amerikanischem Recht würde diese Begriffsverwendung freilich mit dem gesetzlich definierten Begriff der Kopie kollidieren, der nach dem Gesagten auf Werkexemplare beschränkt ist; 17 U.S.C. § 101. immer reproduzierbar gewesen sei und dass alles, was Menschen gemacht hatten, immer schon "Von Menschen nachgemacht werden" konnte. ${ }^{14}$ Hier stellt Benjamin die Unterscheidung zwischen Kunstwerk (Original) und Reproduktion (Kopie) ausdrücklich in den Kontext des Nachmachens, was in urheberrechtlicher Hinsicht zweifellos ein Thema ist, das die Werkseite der Leitdifferenz betrifft. Zumindest aus schweizerischer Perspektive ist die Formulierung Benjamins ein geradezu untrügliches Zeichen für dieses Verständnis, denn im schweizerischen Immaterialgüterrecht ist ,Nachmachen' der tradierte Fachbegriff für identisches Kopieren (im Unterschied zur veränderten Übernahme eines vorbestehenden Werks, die als ,Nachahmung' bezeichnet wird). ${ }^{15}$

Zwar ist diese Verwendung der Unterscheidung von Original und Kopie für das moderne Urheberrecht ungewohnt, aber das damit beschriebene Konzept ist materiell bestens bekannt, denn es geht letztlich um nichts anderes als die klassische Urheberrechtsverletzung. Wer ein urheberrechtlich geschütztes Werk ohne Zustimmung des Berechtigten nachmacht oder nachahmt, greift in das ausschließliche Recht des Urheberrechtsinhabers ein und begeht eine Urheberrechtsverletzung, solange er sich dafür nicht auf eine gesetzliche Ausnahme stützen kann. Der Grund, weshalb im modernen Urheberrecht zur Beschreibung dieses Sachverhalts nicht auf die Unterscheidung von Original und Kopie zurückgegriffen wird, liegt wohl primär darin, dass die Gesetze diese Begriffe in diesem Zusammenhang nicht verwenden. In der Tat wird der Begriff des Originals, so er denn in der Urheberrechtssprache überhaupt vorkommt, nicht als Synonym für Werke im Allgemeinen gebraucht, sondern zur Bezeichnung ganz bestimmter Werkexemplare. ${ }^{16}$ Damit ist zugleich die andere Seite der urheberrechtlichen Leitdifferenz angesprochen.

14 Benjamin 1991, S. 474: „Das Kunstwerk ist grundsätzlich immer reproduzierbar gewesen. Was Menschen gemacht hatten, das konnte immer von Menschen nachgemacht werden."

15 Siehe z.B. Art. 66 lit. a des schweizerischen Patentgesetzes (PatG) und Art. 61 Abs. 1 des schweizerischen Markenschutzgesetzes (MSchG).

16 Siehe z.B. Dreier 2016, S. 255: "the original can be described as the work's first reproduction"; terminologisch unpräzise daher Schack 2017, Rz. 22, S. 17: "Original ist allein das ursprüngliche Werk." 


\section{Original als Originalwerkexemplar?}

Wechselt man auf die andere Seite der urheberrechtlichen Leitdifferenz, dann bezeichnen die Begriffe des Originals und der Kopie nicht mehr verschiedene Werke als geistige Schöpfung, sondern unterschiedliche Exemplare desselben Werks. Der Begriff des Originals wird in diesem Fall zur Kurzform des Ausdrucks ,Originalwerkexemplar', ${ }^{17}$ während der Begriff der Kopie für ein beliebiges anderes Werkexemplar steht, das man im deutschsprachigen Raum in der Regel ,Vervielfältigungsstück' nennt. ${ }^{18}$ Dabei spielt es auf dieser Seite der Leitdifferenz juristisch keine Rolle, ob das in Frage stehende Vervielfältigungsstück vom Urheberrechtsinhaber oder von einem Dritten hergestellt wurde.

Dieses Begriffsverständnis passt am besten auf das moderne Urheberrecht, und um dieses Verständnis scheint es auch Walter Benjamin gegangen zu sein, als er schrieb, dass auch "bei der höchstvollendeten Reproduktion" eines ausfalle, nämlich "das Hier und Jetzt des Kunstwerks - sein einmaliges Dasein an dem Orte, an dem es sich befindet". ${ }^{19}$ Da Werke im Sinne von geistigen Schöpfungen ubiquitär sind und sich daher definitionsgemäß nicht geografisch loka-

17 Setzt man - inspiriert vom amerikanischen Recht Werkexemplare mit Kopien (,copies') gleich, dann könnte man auch von ,Originalkopie' sprechen, doch würde dies in der deutschen Rechtssprache nur Verwirrung stiften. Im Übrigen ist der Begriff der ,Originalkopie' bereits anderweitig belegt, nämlich als "erste Kopie des Originals", die unter bestimmten Voraussetzungen die "Funktion eines Originals" annehmen kann; Weingart 2012, S. 203. Aus urheberrechtlicher Sicht müsste man hier freilich von der ,ersten Kopie des Originalwerkexemplars' sprechen.

18 Eine stärker differenzierende Terminologie findet sich in einem Urteil des schweizerischen Bundesgerichts von 1942, in dem es - abgesehen vom Werk als Immaterialgut - vom "Werkgegenstand" als "Verkörperung des Werks" und von "Werkexemplaren" als "Vervielfältigungen des Werkgegenstandes" sprach; BGE 68 III 65, E. 2. Es besteht indes kein Grund, neben Werk und Werkexemplar noch einen ,Werkgegenstand' anzuerkennen, denn entweder meint man damit ein verkörpertes Werk (dann geht es eben um ein Werk) oder man meint eine spezifische Art von Werkexemplar, also z.B. Vervielfältigungsstücke im Unterschied zu Originalwerkexemplaren (dann geht es eben um Werkexemplare in dem Sinne, wie der Begriff in diesem Beitrag verstanden wird).

19 Benjamin 1991, S. 475. lisieren oder gar an einem bestimmten physischen Ort festmachen lassen, kann Benjamin mit dieser Passage nur Werkexemplare gemeint haben. Der Gegenüberstellung von Kunstwerk (Original) und Reproduktion (Kopie) lässt sich im vorliegenden Kontext weiter entnehmen, dass er mit ,Kunstwerk' an dieser Stelle wohl an das ursprüngliche Werkexemplar dachte, das vom Urheber eigenhändig geschaffen wurde und das überdies auch ein Unikat ist. Wäre Walter Benjamin Urheberrechtler gewesen, so hätte er hier von ,Originalwerkexemplar' statt von ,Kunstwerk' gesprochen.

Während Benjamin hinsichtlich der Unschärfe seiner Diktion in juristischer Hinsicht sicher kein Vorwurf gemacht werden kann, so gilt dies nicht gleichermaßen für den schweizerischen Gesetzgeber, von dem man eine saubere terminologische Unterscheidung zwischen Werk und Werkexemplar durchaus hätte erwarten können. Die Rede ist von einer gesetzlichen Bestimmung, bei der es inhaltlich gerade um die Unterscheidung zwischen Urheberrecht am Werk und Eigentum am Werkexemplar geht und die wie folgt lautet:

Die Übertragung des Eigentums am Werkexemplar schliesst urheberrechtliche Verwendungsbefugnisse selbst dann nicht ein, wenn es sich um das Originalwerk handelt. ${ }^{20}$

In der Sache will diese Norm sagen, dass die Übertragung des Eigentums an einem Werkexemplar die Übertragung des Urheberrechts am darin verkörperten Werk nicht mit einschließt. Dies ist an sich selbstverständlich, weil es sich dabei nach dem Gesagten um unterschiedliche Rechtsobjekte und unterschiedliche Rechte handelt. Bei Massenware wie Büchern leuchtet diese Regel auch unmittelbar ein, denn wer ein Werkexemplar zu Eigentum erwirbt, ist sich bewusst, dass damit nicht zugleich der Erwerb des Urheberrechts am literarischen Werk einhergeht. Beim Verkauf von Unikaten im Bereich der bildenden Kunst wurde im 19. Jahrhundert jedoch darüber gestritten, ob mit der physischen Übergabe des Unikats zu Eigentum zugleich auch über das Vervielfältigungsrecht am darin ver-

20 Art. 16 Abs. 3 URG. 
körperten Kunstwerk verfügt wird, ${ }^{21}$ zumal der Urheber nach Übergabe des Unikats nicht mehr ohne weiteres in der Lage ist, sein Urheberrecht am verkörperten Werk selbst auszuüben. Um in dieser Hinsicht Klarheit zu schaffen, hat der schweizerische Gesetzgeber die besagte Norm ins Urheberrechtsgesetz aufgenommen. ${ }^{22}$ Sie schreibt explizit vor, dass die Übertragung des Eigentums am Werkexemplar die Übertragung des Urheberrechts selbst dann nicht einschließt, wenn es sich um das "Originalwerk" handelt. ${ }^{23}$ Gemeint ist hier aber offensichtlich nicht das Werk als geistige Schöpfung, sondern das Originalwerkexemplar, also das Kunstwerk im Sinne Walter Benjamins.

Für die Zwecke dieses Beitrags ist diese Gesetzesbestimmung - abgesehen von ihrem Illustrationswert für den Mangel an sprachlicher Präzision - indes nicht besonders ergiebig, auch wenn sie den Begriff des Originals ausdrücklich verwendet. Inhaltlich wird mit dieser Norm ja nur verdeutlicht, dass die getroffene Regel sowohl für Vervielfältigungsstücke als auch für Originalwerkexemplare gilt. Mit anderen Worten wird also gerade nicht danach unterschieden, ob das eine oder das andere vorliegt, was dazu führt, dass sich in der Praxis die Abgrenzungsfrage gar nie stellt. In der Sache wesentlich aufschlussreicher sind daher gesetzliche Regeln, bei denen es gerade darauf ankommt, ob ein Originalwerkexemplar oder ein Vervielfältigungsstück vorliegt, denn dann bedingt die Anwendung der betreffenden Regel, dass diese Begriffe trennscharf definiert werden (und zwar spätestens durch die Gerichte im Streitfall). Solche Regeln sind im Urheberrecht zwar selten, aber es gibt sie. ${ }^{24}$ Zwei dieser Regeln - eine aus dem Recht der Europäischen Union, die andere aus dem schweizerischen Recht - sollen nachstehend etwas näher betrachtet werden.

21 Siehe dazu aus historischer Perspektive z.B. die Ausführungen in der „Botschaft des Bundesrathes an die Bundesversammlung, betreffend den Entwurf zu einem Geseze über das Urheberrecht an Werken der Literatur und Kunst" vom 9. Dezember 1881, BBI. 1881 IV 645, S. 651-654.

22 Dies geschah im Kern bereits im ersten schweizerischen Urheberrechtsgesetz von 1881 (dort Art. 5 Abs. 1) und fand sich auch im zweiten Urheberrechtsgesetz von 1922 (dort Art. 9 Abs. 3).

23 Art. 16 Abs. 3 URG.

24 Siehe auch Bullinger 2006, S. 106-112; Schack 2017, Rz. 28-29, S. 20-21.

\section{Urheberrechtliche Relevanz I: Folgerecht}

Ein Bereich des Urheberrechts, in dem mit der Unterscheidung zwischen Original und Kopie auf der Ebene von Werkexemplaren operiert wird, ist das Folgerecht (,resale right'), dessen Anerkennung den Mitgliedstaaten der Europäischen Union in einer Richtlinie ${ }^{25}$ vorgeschrieben wird. ${ }^{26}$

Das Folgerecht ist das Recht bestimmter bildender Künstler auf Beteiligung am Verkaufserlös aus jeder Weiterveräußerung von Originalwerkexemplaren, die nach der Erstveräußerung durch den Urheber über den Kunstmarkt erfolgt. ${ }^{27} \mathrm{Hin}$ tergrund dieser Regelung ist, dass man bildende Künstler an der Wertsteigerung von Originalwerkexemplaren beteiligen will. Die Ausgangslage dafür ist nach allgemeinem Urheberrecht zunächst jedoch denkbar ungünstig, denn grundsätzlich gilt die Regel, dass Urheber die Weiterverbreitung der von innen selbst in Verkehr gebrachten Werkexemplare nicht kontrollieren können. Wenn also eine Künstlerin ein bestimmtes Werkexemplar an einen Kunsthändler verkauft, dann sind ihre Verbreitungsrechte an diesem konkreten Exemplar ,erschöpft', mit der Folge, dass der Kunsthändler dieses Exemplar weiterverkaufen darf, ohne dass dies von der Künstlerin verboten oder an Bedingungen geknüpft werden könnte. Dies ist an sich richtig, denn das betreffende Exemplar befindet sich im Eigentum des Kunsthändlers und die Künstlerin hatte anlässlich des Erstverkaufs bereits die Gelegenheit, eine ihr angemessen erscheinende Vergütung zu erzielen. Umgekehrt impliziert dies, dass die Künstlerin leer ausgeht, wenn der Kunsthändler beim Weiterverkauf einen Erlös erzielt, und zwar auch dann, wenn der Wert des veräußerten Werkexemplars in der Zwischenzeit erheblich gestiegen sein sollte. Das Folgerecht der EU setzt an diesem Punkt an und ändert die allgemeine urheberrechtliche Regel teilweise, indem Urhebern bestimmter Kunstwerke ein Anspruch auf Beteiligung am

25 Richtlinie 2001 / 84 / EG des Europäischen Parlaments und des Rates vom 27. September 2001 über das Folgerecht des Urhebers des Originals eines Kunstwerks, ABI. EG L 272 vom 13.10.2001, S. 32-36 (hiernach „Folgerechts$\left.\mathrm{RL}^{\prime \prime}\right)$.

26 Siehe Art. 1 Abs. 1 Folgerechts-RL.

27 Art. 1 Abs. 1 und 2 Folgerechts-RL. 
Verkaufserlös gewährt wird, sofern der Weiterverkauf über den Kunstmarkt erfolgt - also unter der Beteiligung von Auktionshäusern, Kunstgalerien oder Kunsthändlern als Käufern, Verkäufern oder Vermittlern. Nach der gesetzlichen Ordnung muss die Folgerechtsvergütung vom Veräusserer bezahlt werden, und zwar unabhängig davon, ob er mit dem Verkauf einen Gewinn erzielt. Im Wesentlichen wirkt das Folgerecht daher wie eine Kunstmarkttransaktionssteuer zugunsten ausgewählter Urheber.

In diesem Zusammenhang musste unter anderem definiert werden, für welche Gegenstände das Folgerecht überhaupt gilt - und da wurde auf den Begriff des "Originals eines Kunstwerks" abgestellt. ${ }^{28}$ Bei der näheren Definition dieses Begriffs orientierte man sich dann freilich nicht am Begriffsverständnis Walter Benjamins, sondern an den Präferenzen des Kunstmarkts. ${ }^{29}$ Entsprechend "gelten" als "Originale von Kunstwerken" im Sinne der Folgerechtsrichtlinie nicht nur "Werke der bildenden Künste", die "vom Urheber selbst geschaffen worden sind", sondern auch Exemplare, "die als Originale von Kunstwerken angesehen werden." ${ }^{\prime 30}$ Dabei fällt zunächst auf, dass die Leitdifferenz von Werk und Werkexemplar terminologisch auch hier nicht vollständig umgesetzt wird, denn trotz der Verwendung des Werkbegriffs geht es in der Sache einzig um Werkexemplare. Sodann geht aus der gesetzlichen Formulierung hervor, dass eigenhändig geschaffene Unikate zwar den Kern des Begriffs ,Originale von Kunstwerken' ausmachen, davon aber auch andere Werkexemplare erfasst werden, also Vervielfältigungsstücke - jedenfalls solange diese unter der Leitung des Künstlers in begrenzter Auflage hergestellt wurden. ${ }^{31}$ Würde man den Originalbegriff auf ,Originale mit Aura' im Sinne Walter Benjamins beschränken, könnte man nur einen kleinen Teil des Kunstmarkts dem Folge-

28 Art. 1 Abs. 1 Folgerechts-RL. In der englischsprachigen Fassung der Richtlinie wird der Begriff, original work of art' verwendet.

$29 \mathrm{Zu}$ diesem Phänomen allgemein Mosimann / Müller-Chen 2011, S. 1303; Bullinger 2006, S. 108-109.

30 Art. 2 Abs. 1 Folgerechts-RL. Die englische Fassung dieser Norm lautet im Wesentlichen wie folgt: "works of graphic or plastic art [...], provided they are made by the artist himself or are copies considered to be original works of art".

31 Art. 2 Abs. 2 Folgerechts-RL. recht unterwerfen, was insofern unbefriedigend wäre, als die Kunstmarktakteure den Originalbegriff längst verwässert haben und diese Entwicklung bei der Regelung der Folgerechts nicht ignoriert werden kann.

In diesem Zusammenhang geht es beim Begriff des Originals denn auch weniger um die Ursprünglichkeit des fraglichen Werkexemplars als um seine ,Echtheit'. ${ }^{32}$ Dieser Aspekt klingt auch bei Walter Benjamin an, wenn er schreibt, dass das "Hier und Jetzt des Originals den Begriff seiner Echtheit" ausmache. ${ }^{33}$ Juristisch geht es dabei jedoch um Fragen der Zuordnung, z.B. ob ein bestimmtes Werkexemplar von einer bestimmten Künstlerin stammt. Hingegen geht es nicht um die Frage, ob das betreffende Exemplar auch die erste Verkörperung des fraglichen Werks und insofern ein ,Original' ist. Wenn Wolfgang Beltracchi das von inm geschaffene Gemälde, Rotes Bild mit Pferden' als Campendonk ausgibt, ${ }^{34}$ dann wird man dem Gemälde die Echtheit absprechen, aber urheberrechtlich ist und bleibt es ein Originalwerkexemplar, auch im strengen Sinne Benjamins. Mit der Unterscheidung zwischen Original und Kopie hat dies freilich nichts mehr zu tun, und das Urheberrecht ist in solchen Fällen auch nur am Rande betroffen. ${ }^{35}$

32 Siehe dazu auch Schack 2017, Rz. 22, S. 17.

33 Benjamin 1991, S. 476.

34 LG Köln, Urteil 110 KLs 17/11 vom 27. Oktober 2011, E. II.11. Zu ähnlichen Fällen älteren Datums, siehe auch Hamann 1949, S. 140, 148.

35 Die falsche Zuordnung eines Werkexemplars ist nämlich nur dann eine Urheberrechtsverletzung, wenn es sich um ein ,Plagiat' im juristischen Sinne handelt, wenn also jemand ein Werk eines Dritten nachmacht und unter eigenem Namen ausgibt. In diesem Fall wird das Recht des (wahren) Urhebers auf Anerkennung seiner Urheberschaft (siehe z.B. Art. 9 Abs. 1 URG; § 13 UrhG) verletzt und überdies auch sein Vervielfältigungsrecht (Art. 10 Abs. 2 lit. a URG; § 16 UrhG). Im umgekehrten Fall, also der klassischen Kunstfälschung, bei der ein Werk von einer Person geschaffen wird, die dann so tut, als ob das Werk von einem Dritten - typischerweise einem anerkannten Künstler - stammt, liegt indes keine Urheberrechtsverletzung vor, auch wenn bestimmte Straftatbestände erfüllt sein mögen (bei falscher Signierung eines Bildes z.B. eine Urkundenfälschung). 


\section{Urheberrechtliche Relevanz II: Zerstörungsschutz}

Die Schweiz kennt zwar kein Folgerecht, hat dafür aber eine Gesetzesnorm, die sich mit dem Schutz bestimmter Werkexemplare vor Zerstörung befasst und in diesem Kontext explizit mit den Begriffen ,Originalwerk' und ,Originalexemplar' operiert. ${ }^{36}$ Die ersten beiden Absätze dieser Norm lauten wie folgt:

Müssen Eigentümer und Eigentümerinnen von Originalwerken, zu denen keine weiteren Werkexemplare bestehen, ein berechtigtes Interesse des Urhebers oder der Urheberin an der Werkerhaltung annehmen, so dürfen sie solche Werke nicht zerstören, ohne dem Urheber oder der Urheberin vorher die Rücknahme anzubieten. Sie dürfen dafür nicht mehr als den Materialwert verlangen.

Sie müssen dem Urheber oder der Urheberin die Nachbildung des Originalexemplars in angemessener Weise ermöglichen, wenn die Rücknahme nicht möglich ist. ${ }^{37}$

Auch hier fällt zunächst auf, dass es der schweizerische Gesetzgeber nicht geschafft hat, begrifflich sauber zwischen Werk und Werkexemplaren zu unterscheiden. In der Sache geht es darum, dass Eigentümer von Originalwerkexemplaren - wie es im ersten Absatz richtig heißen sollte - unter bestimmten Umständen die gesetzliche Pflicht trifft, ihre Zerstörungsabsicht den Urhebern mitzuteilen, um ihnen die Rücknahme oder die Nachbildung zu ermöglichen. Die Zerstörung von Originalwerkexemplaren ist demnach urheberrechtlich nicht schlechthin verboten, ${ }^{38}$ sondern (nur, aber immerhin) unter bestimmten Bedingungen zulässig. Dies liegt daran, dass es bei dieser Regel nicht etwa um Denkmalschutz im öffentlichen Interesse geht, sondern um den weniger absoluten Schutz des privaten Erhaltungsinteresses der Urheber. $^{39}$

Für die Zwecke des vorliegenden Beitrags steht im Vordergrund, dass es für die Anwendbarkeit dieser Norm entscheidend darauf ankommt, ob jemand Eigentümerin oder Eigentümer eines Originalwerkexemplars ist. Im Unterschied zur

36 Art. 15 URG.

37 Art. 15 Abs. 1 und 2 URG.

38 Anders das amerikanische Recht bei ,works of recognized stature'; siehe 17 U.S.C. § 106A(a)(3)(B).

39 Siehe Rigamonti 2013, S. 331-332.
Rechtslage beim Folgerecht liefert das Gesetz keine nähere Definition dieses Begriffs, aber es ist gemeinhin anerkannt, dass es sich dabei um die erste Verkörperung des vollständigen Werks handelt. ${ }^{40}$ Immerhin lassen sich dem Normtext zwei wichtige Punkte entnehmen, die vor dem Hintergrund des Kunstwerkverständnisses Benjamins besonders aufschlussreich sind. Erstens schützt diese Regel nicht einfach das letzte existierende Werkexemplar, ${ }^{41}$ denn der Schutz greift nur, wenn das letzte existierende Werkexemplar zugleich auch das originale Werkexemplar ist. Der schweizerische Gesetzgeber macht hier also bewusst einen Unterscheid zwischen schützenswerten Originalwerkexemplaren und nicht schützenswerten Vervielfältigungsstücken. ${ }^{42}$ Zweitens werden auch Originalwerkexemplare nicht ausnahmslos geschützt, sondern eben nur dann, wenn sie Unikate sind, ${ }^{43}$ wenn im Zeitpunkt der beabsichtigten Zerstörung durch den Eigentümer also keine weiteren Exemplare des betreffenden Werks bestehen. ${ }^{44}$ Gibt es solche weiteren Exemplare, dann darf der Eigentümer sogar das Originalwerkexemplar vernichten, ohne dass er seine Zerstörungsabsicht dem Urheber mitteilen müsste.

Im Ergebnis mag das Zusammenspiel dieser zwei Punkte auf den ersten Blick etwas merkwür-

40 Siehe z.B. Rigamonti 2013, S. 334. Im Einzelfall ist freilich nicht immer klar, was ein Originalwerkexemplar ist, insbesondere im Bereich der Fotografie und des digitalen Werkschaffens; vgl. dazu Mosimann / Müller-Chen 2011, S. 1304-1312; Hamann 1981, S. 45-48; Bullinger 2006, S. 106-112.

41 Kritisch diesbezüglich Zech / Anger 2016, S. 1166-1167. 42 Dazu passt auch die Formulierung des Bundesgerichts im bereits erwähnten Urteil von 1942 (oben Fn. 18), das wenn auch in anderem Kontext - das Folgende festhielt: "es ist eine Eigenart der Malerei, dass neben dem Originalwerk weitern Werkexemplaren keine nennenswerte Bedeutung zukommt"; BGE 68 III 65, E. 2.

43 Die französischsprachige Gesetzesfassung von Art. 15 Abs. 1 URG, die nach schweizerischem Verständnis ebenso authentisch ist wie die deutschsprachige (Art. 14 Abs. 1 PublG), zeigt dies besonders deutlich, denn dort ist ausdrücklich von "I'unique exemplaire original" die Rede.

44 Man könnte sich hier freilich fragen, ob mit dem Ausdruck ,keine weiteren Werkexemplare' jedwelche Vervielfältigungsstücke gemeint sind, wie es der Wortlaut von Art. 15 Abs. 1 URG suggeriert und wie dies in der Schweiz traditionell verstanden wird, oder ob damit nach dem Zweck dieser Bestimmung nur solche mit Originalqualität erfasst werden sollten, wie man sie z.B. aus der seriellen Kunst kennt. 
dig erscheinen, ist aber gerade vor dem Hintergrund von Walter Benjamins Vorstellung von der ,Aura' des einmaligen Kunstwerks eigentlich nur konsequent. Diejenigen Urheber, die nicht nur Unikate schaffen, sondern der Versuchung erliegen, die technischen Möglichkeiten zu nutzen und ihre Werke durch die Herstellung von Reproduktionen zur ,aurafreien' Massenware zu machen, werden nur in einem reduzierten Masse geschützt - nämlich nur dann, wenn das Originalwerkexemplar, das zerstört werden soll, zugleich auch das letzte bestehende Werkexemplar ist. Ansonsten ist es das Originalwerkexemplar offenbar nicht wert, erhalten zu werden. Auch wenn wohl ohne weiteres ausgeschlossen werden kann, dass sich der schweizerische Gesetzgeber beim Erlass dieser Gesetzesnorm an Walter Benjamin orientierte, so ist doch bemerkenswert, dass es gerade seine Vorstellung von der auratischen Natur des unikalen Kunstwerks ist, die eine tiefere Begründung für die genannte Gesetzesnorm zu liefern vermag. Darin liegt zugleich die bleibende praktische Bedeutung Walter Benjamins für das schweizerische Urheberrecht.

\section{Fazit}

Als Fazit lässt sich festhalten, dass die Unterscheidung zwischen ,Original' und ,Kopie' oder - in der Diktion Benjamins - zwischen ,Kunstwerk' und ,Reproduktion' im modernen Urheberrecht unterschiedliche Bedeutungen haben kann, die letztlich wenig überraschend vom juristischen Kontext bestimmt werden, in dem die Unterscheidung zur Anwendung gelangt. Der Anwendungsbereich dieser Unterscheidungen ist freilich insofern beschränkt, als die urheberrechtliche Leitdifferenz eben nicht die Unterscheidung zwischen Original und Kopie, sondern die Unterscheidung zwischen Werk und Werkexemplar ist. Dennoch gibt es heute sowohl in der Europäischen Union als auch in der Schweiz Gesetzesnormen, die entscheidend darauf abstellen, ob ein Originalwerkexemplar vorliegt - und in diesem Kontext leben auch die Vorstellungen von Walter Benjamin teilweise noch fort.

\section{Literaturverzeichnis}

Benjamin, Walter (1991 [1936]): Das Kunstwerk im Zeitalter seiner technischen Reproduzierbarkeit. In: Benjamin, Walter: Gesammelte Schriften. Bd. I/2. Hg. von Tiedemann, Rolf / Schweppenhäuser, Hermann. Frankfurt a. M.: Suhrkamp, S. 471-508.

Bullinger, Winfried (2006): Urheberrechtlicher Originalbegriff und digitale Technologien. In: Kunst und Recht. Journal für Kunstrecht, Urheberrecht und Kulturpolitik (KUR), 8/4, S. 106-112.

Dreier, Thomas (2016): The Ethics of Copyright and droit d'auteur. An Outline. In: Hick, Darren Hudson / Schmücker, Reinhold (Hgg.): The Aesthetics and Ethics of Copying. London: Bloomsbury, S. 251-269.

Dreier, Thomas (2017): Bilder im Zeitalter ihrer vernetzten Kommunizierbarkeit. In: Zeitschrift für Geistiges Eigentum (ZGE), 9/2, S. 135-148.

Jayme, Erik (2016): Nachahmung oder Transformation: Zweitkunst im Zwielicht des Rechts. In: Mosimann, Peter / Schönenberger, Beat (Hgg.): Kunst \& Recht. Bd. 8. Bern: Stämpfli, S. 14-38.

Hamann, Richard (1949): Original und Kopie. In: Marburger Jahrbuch für Kunstwissenschaft, Bd. 15, S. $135-156$.

Hamann, Wolfram (1981): Grundfragen der Originalfotografie. In: Archiv für Urheber-, Film-, Funk- und Theaterrecht (UFITA), 90, S. 45-58.

Luhmann, Niklas (1987): Soziale Systeme. Grundriss einer allgemeinen Theorie. Frankfurt a. M.: Suhrkamp.

Mosimann, Peter / Müller-Chen, Markus (2011): Kauf eines Originals der bildenden Kunst, insbesondere einer Fotografie. In: Büchler, Andrea / Müller-Chen, Markus (Hgg.): Festschrift für Ingeborg Schwenzer. Bd. 2. Bern: Stämpfli, S. 1303-1323.

Rehbinder, Manfred (2000): Schweizerisches Urheberrecht. 3. Auflage. Bern: Stämpfli.

Rigamonti, Cyrill P. (2013): Urheberpersönlichkeitsrechte. Bern: Stämpfli.

Schack, Haimo (2017): Kunst und Recht. 3. Auflage. Tübingen: Mohr Siebeck.

Schöttker, Detlev (2015): Kommentar. In: Benjamin, Walter: Das Kunstwerk im Zeitalter seiner technischen Reproduzierbarkeit und weitere Dokumente. 4. Auflage. Frankfurt a. M.: Suhrkamp, S. 99-254.

Weingart, Brigitte (2012): Originalkopie. In: Bartz, Christina / Jäger, Ludwig / Krause, Marcus / Linz, Erika (Hgg.): Handbuch der Mediologie. Paderborn: Fink, S. 203-208.

Zech, Herbert / Anger, Christian (2016): Die Zerstörung urheberrechtlich geschützter Werke. In: Fankhauser, Roland / Widmer Lüchinger, Corinne / Klingler, Rafael et al. (Hgg.): Das Zivilrecht und seine Durchsetzung, Festschrift für Thomas Sutter-Somm. Zürich: Schulthess, S. 1149-1168. 